\title{
Fungus infected atrial myxoma: A rare cause of infective endocarditis and chordae rupture
}

\author{
Gökhan Özmen ${ }^{1}$, Kübra Doganay², Hasan Ari $^{3}$, Tolga Dasli ${ }^{4}$ Ahmet Tutuncu ${ }^{5}$, Mehmet Melek \\ Mehmet Demir ${ }^{7}$, Erhan Tenekecioglu ${ }^{8}$
}

1,2,3,5,6,7,8 Bursa Yüksek Ihtisas Heart Education and Research Hospital, ${ }^{4}$ Kocaeli Derince Education and Research Hospital

Case reports are increasing about infected myxomas. Infection with bacteria is relatively much more but fungus infected atrial myxomas are exteremely rare and may complicate the disease course. As our knowledge this is the first fungus infected myxoma report in Turkish population.

Key words: Atrial myxoma, Fungus, Infective endocarditis, Chordae rupture
Access this article online

Website:

http://nepjol.info/index.php/AJMS

DOI: 10.3126/ajms.v6i3.11045

\section{INTRODUCTION}

Atrial myxoma is the most frequently occurring primary tumor of the heart originating from the endocardium as a pedunculated mass. ${ }^{1}$ In a very few cases, this benign neoplasm becomes infected, manifesting itself with symptoms of fever or sepsis with unclear origin. Cardiac myxomas may simulate infective endocarditis but are rarely actually infected. Accurate diagnosis has consistently increased since the introduction of transthoracic echo and solely achieved by surgery. ${ }^{2}$ If surgery is not managed, systemic or cerebral embolization may be unavoidable. Rarely, patients develop unusual symptoms that complicate the diagnostic evaluation. Absolute diagnose of infected myxoma needs clinic and pathologic findings. Here, we report a case who was diagnosed with histopathologically fungus infected left atrial myxoma that was initially appear to be complicated by concomitant infective endocarditis of mitral valve and chorda rupture.

\section{CASE REPORT}

A 70 year old male patient referred to our hospital with fever of unknown origin, mitral regurgitation and dyspnea. On history he has no co-morbid condition or any chronic disesase, lives in a small town and working in farming. Two weeks earlier he recieved antibiotic therapy for history of pneumonia and bilateral pleural effusion. Pneumonia etiology is unknown. He was complainting about intermittent fever, night sweats, fatigue and malaise. Though he had taken this therapy for one week at other hospital fever did not resolve. Consequently he referred to a cardiologist and transthoracic echocardiography had revealed moderate mitral regurgitation with a cardiac mass in left atrium.

On admission he seemed pallor, showed tachypnea, temperature of $38^{\circ} \mathrm{C}$, blood pressure was $110 / 70 \mathrm{mmHg}$ and pulse was 110 beats per minute. Heart sounds were rhytmic, grade $3 / 6$ pansistolic murmur was noted at the cardiac apex. Any pretibial edema were not seen. ECG revealed sinus tachycardia. Posterior-Anterior view of lung graphy showed increased vascularity and uncertain consolidation at upper lobes thought possibility of pulmoner edema may be underlying pneumunia. Laboratory investigation revealed WBC $15.1 \times 10^{3} / \mu \mathrm{l}$, PLT counts 133000, C-reactive protein $119 \mathrm{mg} / \mathrm{L}$, sedimentation rate was $96 \mathrm{~mm}$. Trans thoracic echocardiography revealed slight degeneration on mitral valve accompanying moderate regurgitation, $1.4 \mathrm{~cm}$ moving free chorda mimics vegetation at atrial side of mitral anterior valve and small masses, concomitant $4 \times 2.5-3 \mathrm{~cm}$ heterogen pediculated moving mass like myxoma in left atrium. All other cardiac chambers size, global ejection fraction and other valvular structures were almost normal. Concurrent transesophageal echo 
confirmed moderate to severe mitral regurgitation with mobile $1.4 \mathrm{~cm}$ mass in atrial face and a small mobile masses were seen in atrial surface. Showed $4.0 \times 3 \mathrm{~cm}$ highly mobile mitral annular touching mass arose from interatrial septum (Figures 1-3).

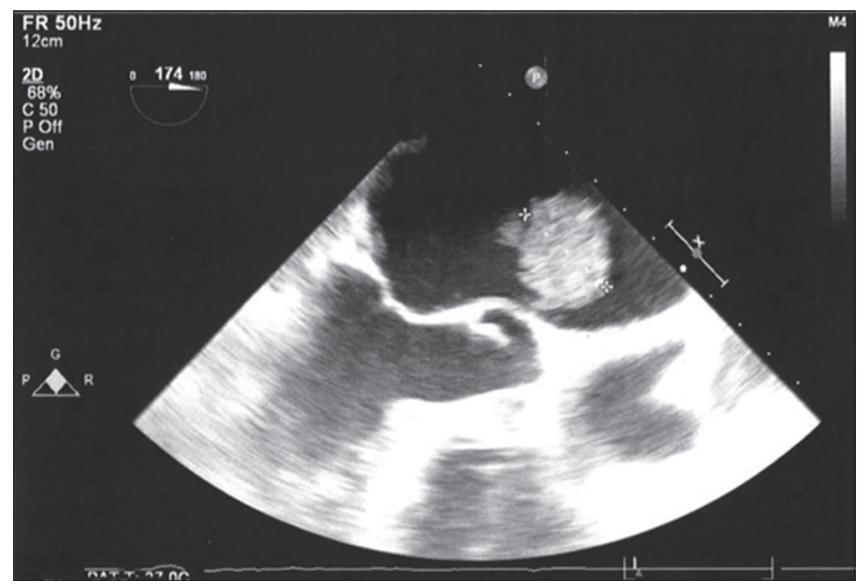

Figure 1: Higly mobile $4 \mathrm{~cm} \times 3 \mathrm{~cm}$ pedunculated left atrium heterogen amorphous mass

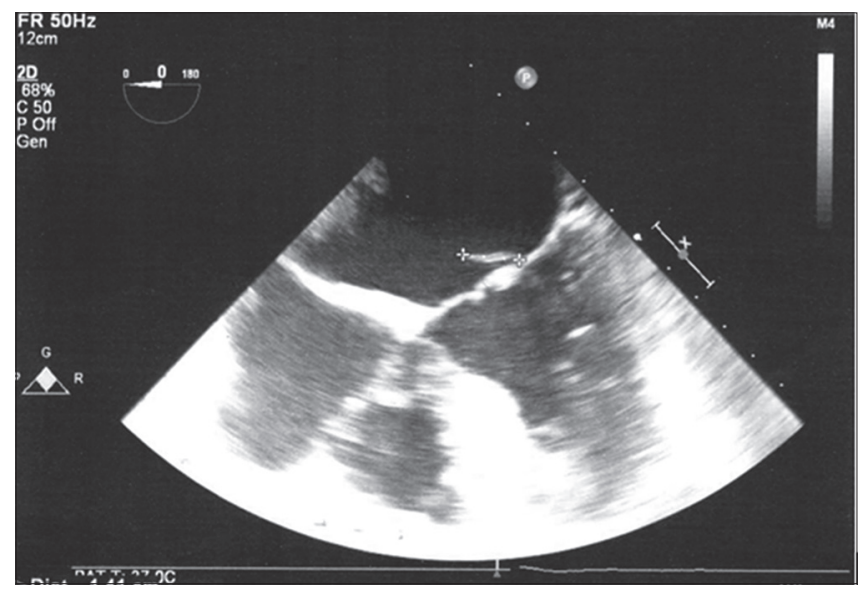

Figure 2: Fibril like mass on left atrial face at four chamber TEE view

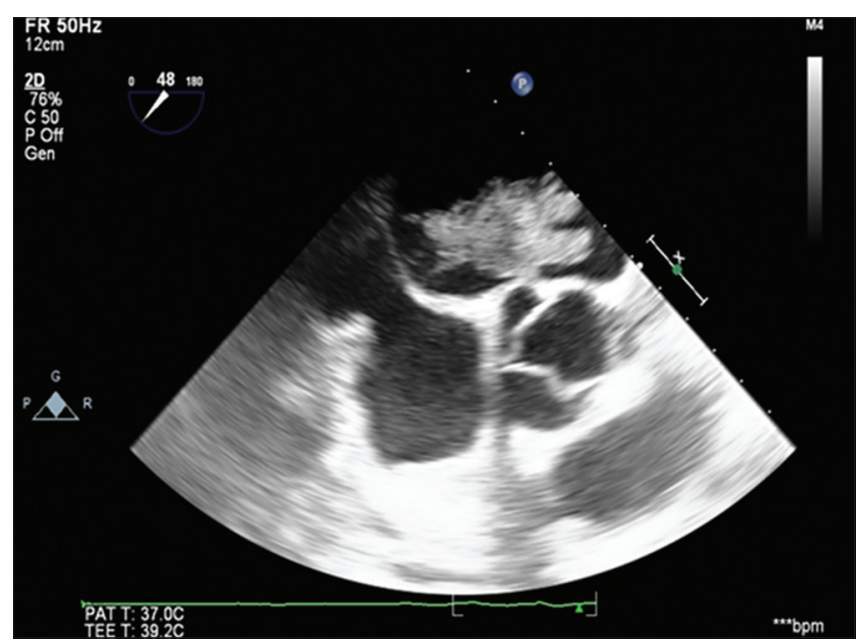

Figure 3: Short axis image of myxoma on TEE examination
For mobile mass at atrial face mitral valve ruptured chordae was thought because of moderate to severe regurtitation. For other small mobile masses we thought as the possibility of vegetation.

Blood cultures were collected in our center had been negative for bacteria and just one of six sample non specific Staphylococcus hominis had grown.

Vascular and immunologic findings for infective endocarditis were not seen. Meanwhile for possibility of infective endocarditis empirical ampisilin-sulbaktam and gentamycin therapy were given and temperature keep on $37^{\circ} \mathrm{C}$. He seemed better and no hemodynamic instability. He seemed better after antibiotic with no fever afterward. Surprisingly patient deteriorated at second week of hospitalization. Fever and dyspnea persisted. Systolic murmur was musical hard than first, ralles were bilateral and lung radiography made us thought pulmoner edema and pneomonia. Transesophageal echo repeated showed ruptured chordae and severe mitral regurtitation. As hemodynamic decompansation and fever antibiotic therapy was switched by linezolid and ciprofloksasin. After hemodynamy stabilized and infection controlled as he is older coronary angiogram performed and revealed three vessel culprit stenosis. Open heart surgery was performed successfully. Left atrial mass excised, mitral valve is replaced by number 23 bilifleat metalic prothesis and at the same time coronary bypass graft was performed. Patient tolerated surgery well and he went on receiving $4 \times 100 \mathrm{mg}$ fluconazol therapy. He was discharged after two weeks without any complication.

\section{DISCUSSION}

Mostly myxomas show atypical symptoms also seen with in large clinical diagnosing spectrum. So appearing of myxomas various and often can be mistaken for symptoms of other conditions. The most common systemic symptoms include fatigability, joint pain, weight loss, and low-grade fever. Myxoma can mimicmitral annular obstruction or lead to mitral regurgitation, thus producing symptoms such as dyspnea, syncope,angina pectoris, peripheral embolism, and heartfailure. ${ }^{3-6}$

Infected cardiac myxoma was first reported in $1956 .{ }^{7}$ Infected cardiac myxoma with coexisting valvular endocarditis has also been reported. ${ }^{8}$ But first fungus infected cardiac myxoma was reported by Joseph at al in $1980 .{ }^{9}$

In the first look mitral vegetative images on atrial face with chordae rupture together with clinical and laboratory findings suggested infective endocarditis. Under antibiotic therapy he worsened and serial blood cultures were 
negative for typical bacterias. Owing to the fact that he received antibiotic therapy cultures may be negative so blood-culture negative infective endocarditis arises most commonly as a consequence of prior antibiotic administration. Hence this might be the reason why the cultures were negative.

As surgical view there were not any evidence of infective tissue but because of chordae rupture and severe mitral regurgitation excision of the valve was inevitable. In our patient, histologic examination did not reveal bacteria; however, the myxomatous tissue showed fungi elements (spores and hyphas) and thrombus composed of fibrin with neutrophils and macrophages, and the finding was confirmed by histopathology. Hence, the diagnosis of fungus infected myxoma was highly probable. Also patients clinical condition supported infective endocarditis. Add to this he recovered dramatically at post op period with coexisting fluconazol therapy. Myxoma related mitral valve endocarditis was possible but after histopathologic examination and surgical view fungus infected left atrial myxoma was not contradictory. Because we could not confirmed infective endocarditis by blood cultures. At our institue further evaluation about fungi species was not possible. Dropping temperature with given antibiotherapy is conflicting. Because of technical inability we could not diagnose any bacterial species on the samples. Both fungi and bacterial endoctrditis may cause this scenario. Possible underlying immune compromise condition fort his patient did not detect.

The treatment for cardiac myxoma is complete surgical resection. In patients that mass impede the circulation (as in blockage of the mitral annulus) should undergo surgery as soon as possible. Myxomas are very friable and have a high potential for embolism. Therefore, early resection is equally crucial in regard to asymptomatic myxomas, whether infected or uninfected. Regardless of infection, myxomas embolize with a similar incidence of approximately $20 \% .{ }^{10}$ Intraoperatively,manipulation of the heart before clamping should be avoided.

\section{CONCLUSION}

When infected myxoma is diagnosed in time and removed without delay, it is an easily treatable disease with excellent patient outcomes. ${ }^{10,11}$ Antifungal therapy should be given. For this case as fungus substances seen, complete recovery obtained by antifungal therapy. Because it contains a potential risk of arterial embolization early diagnosis and urgent surgery should be considered. Our patient recovered completely. ${ }^{12}$

\section{REFERENCES}

1. Yazici M, Ozhan $\mathrm{H}$, Tetik $\mathrm{O}$, Kinay $\mathrm{O}$ and Ergene $\mathrm{O}$. Isolated large right atrial myxoma manifested by syncope. Tex Heart Inst J 2004;31:324-325.

2. Reynen K. Cardiac myxomas. N. Engl. J. Med. 1995; 333:16101617.

3. Schaff HV and Mullany CJ. Surgery for cardiac myxomas. SeminThorac Cardiovasc Surg 2000;12(2):77-88.

4. Tillmanns H. Clinical aspects of cardiac tumors. Thorac CardiovascSurg 1990;38 Suppl 2:152-156.

5. Zaviacic $L$ and Zaviacic L. Left atrial myxoma. Cor et Vasa 2007;49(7-8):250-253.

6. Janion $M$, Sielski $J$ and Ciuraszkiewicz K. Sepsis complicatinggiant cardiac myxoma. Am J Emerg Med 2008;26(3):387.e3-4.

7. Dick HJ and Mullin EW. Myxoma of the heart complicated by bloodstream infection by Staphylococcus aureus and Candidaparapsilosis. N Y State J Med 1956;56(6):856-859.

8. Vogt PR, Jenni R and Turina MI. Infected left atrial myxoma with concomitant mitral valve endocarditis. Eur J Cardiothorac Surg 1996;10(1):71-73.

9. Joseph P, Himmelstein DU, Mahowald JM, Stullman WS. Atrial myxoma infected with Candida: first survival. Chest 1980;78(2):340-343.

10. Revankar SG and Clark RA. Infected cardiac myxoma. Case report and literature review. Medicine (Baltimore) 1998;77(5):337-344.

11. Riad MG, Parks JD, Murphy PB and Thangathurai D. Infectedatrial myxoma presenting with septic shock. J CardiothoracVasc Anesth 2005;19(4):508-511.

12. Nagata T, Totsuqawa T, Katayama K, Kuinose M, Yoshitaka H and Uesuqi T. Infected cardiac myxoma. J Card Surg 2013;28(6):682-684. 